\title{
PROBLEMAS EN EL ANÁLISIS DE METILSILOXANOS VOLÁTILES (VMS): ORIGEN Y SOLUCIONES
}

\author{
E.Y. Companioni-Damas \\ Departamento de Química Analítica, Universidad de Barcelona, Avenida Diagonal 647, 08028 Barcelona, España
}

Recebido em 24/05/2016; aceito em 09/08/2016; publicado na web em 02/09/2016

\begin{abstract}
PROBLEMS IN THE ANALYSIS OF VOLATILE METILSILOXANES (VMS): ORIGIN AND SOLUTIONS. Volatile methylsiloxanes (VMS) are pollutants widely distributed in the environment due to its extensive uses and high volatility. The analysis of these compounds is commounly performed by gas chromatography - mass spectrometry (GC-MS), and it is dificult due to the presence of background contamination and volatile losses. Different preconcentration techniques such as purge and trap (P\&T), headspace (HS) and headspace - solid phase microextraccion (HS-SPME) have been applied for the analysis of VMS in environmental samples. Alternatively, solvent extraction combined with large volume injection techniques has been an effective method for the analysis of complex matrices (eg. wastewater, sludges). In this review, the efficiency of the different methods reported for the analysis of VMS is discussed, and different solutions are proposed to minimize background contamination and volatilization.
\end{abstract}

Keywords: volatile methylsiloxanes; GC-MS, background contamination; large volume injection.

\section{INTRODUCCIÓN}

Los siloxanos son compuestos constituidos por átomos de silicio y oxígeno enlazados entre sí de forma alternada, donde cada átomo de silicio se encuentra a su vez unido a uno o diferentes grupos orgánicos. Dentro de esta familia de compuestos los metilsiloxanos de cadena corta conocidos como metilsiloxanos volátiles (VMS), han recibido una especial atención en los últimos años debido a su elevada capacidad de bioacumulación, ${ }^{1-7}$ potencial cancerogenicidad ${ }^{8-10}$ y amplia distribución ambiental. ${ }^{11-21}$ Estos compuestos se presentan en conformaciones lineales o cíclicas tal y como se ilustra en la Figura 1, y se caracterizan principalmente por presentar una elevada presión de vapor $\left(2.3-5620 \mathrm{~Pa}\right.$ a $\left.25^{\circ} \mathrm{C}\right)$ y baja solubilidad en agua $\left(0.07-1560 \mu \mathrm{g} \mathrm{L}{ }^{-1}\right.$ a $\left.25^{\circ} \mathrm{C}\right) .{ }^{22-24}$ Entre sus principales aplicaciones se encuentra su uso como constituyentes básicos en cosméticos y productos de higiene personal, como materiales de partida para la síntesis de polisiloxanos de elevado peso molecular, y su empleo como aditivos en productos de limpieza, desengrasantes, lubricantes, disolventes, pinturas, lacas y barnices.

Como consecuencia de su amplia utilización los VMS se emiten en grandes cantidades al medio ambiente. La elevada volatilidad de estos compuestos condiciona que más del $90 \%$ de los VMS se emitan a la atmosfera, mientras que tan solo un $10 \%$ se descarga en las aguas residuales tanto industriales como urbanas. ${ }^{25} \mathrm{Se}$ ha demostrado que su mayor emisión a la atmósfera se produce en zonas cercanas a plantas depuradoras de aguas residuales, aunque también constituyen importantes focos de emisión de VMS, aquellos relacionados al uso y la fabricación de productos de higiene personal, siendo considerados como una de sus principales vías de entrada al medio ambiente. ${ }^{11-13,26}$

En cuanto a la toxicidad de los VMS, la mayor parte de los estudios publicados están enfocados a los VMS cíclicos, ya que este grupo de compuestos son los que se encuentran en mayores concentraciones en el medio ambiente. Los resultados que estos estudios muestran son, en algunos casos, contradictorios. Así por ejemplo, la Agencia de Protección Ambiental de Canadá reporta que el D4 y el D5 entran al medio ambiente en unas concentraciones que pueden causar daños ecológicos. ${ }^{26}$ Sin embargo en otros trabajos se indica que el D5 solo

*e-mail: elocompa@gmail.com

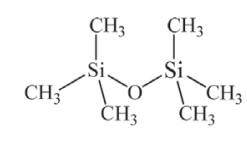

L2: hexametildisiloxano<smiles>C[Si](C)(C)O[Si](C)(C)O[Si](C)(C)C</smiles>

L3: octametiltrisiloxano<smiles>C[Si]1(C)O[Si](C)(C)O[Si](C)(C)O1</smiles>

D3: hexametilciclotrisiloxano<smiles>C[Si]1(C)O[Si](C)(C)O[Si](C)(C)O[Si](C)(C)O[Si](C)(C)O1</smiles>

D5: decametilciclopentasiloxano<smiles>C[Si](C)(C)O[Si](C)(C)O[Si](C)(C)O[Si](C)(C)C</smiles>

L4: decametiltetrasiloxano<smiles>C[Si](C)(C)O[Si](C)(C)O[Si](C)(C)O[Si](C)(C)O[Si](C)(C)C</smiles>

L5: dodecametilpentasiloxano<smiles>C[Si]1(C)O[Si](C)(C)O[Si](C)(C)O[Si](C)(C)O1</smiles>

D4: octametilciclotetrasiloxano

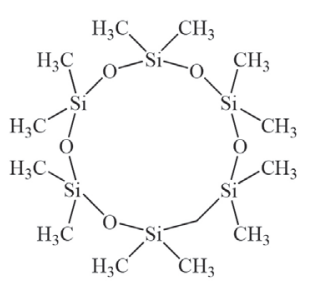

D6: dodecametilciclohexasiloxano
Figura 1. Estructura molecular de los VMS lineales y cíclicos

presenta toxicidad para los organismos que habitan en los sedimentos, donde los VMS tienden a acumularse debido a su elevado carácter hidrofóbico. ${ }^{27}$ Así mismo, el D4 como el D5 han sido clasificados por agencias regulatorias Europeas como compuestos muy bioacumulables, sin embargo al D6 no se le ha dado esta clasificación. ${ }^{28}$ Por su parte, la Agencia de Protección Ambiental de Estados Unidos (EPA) ha incluido al D4 una la lista de compuestos para evaluar su posible regulación ambiental. ${ }^{29}$ Como consecuencia de esta divergencia de 
criterios acerca de la toxicidad y bioacumulación de los VMS, aún no se han establecido regulaciones acerca de su producción y uso.

En relación a los métodos de análisis que se han publicado para determinar VMS, debe decirse que la técnica que se emplea con más frecuencia es la cromatografía de gases acoplada a la espectrometría de masas (GC-MS), utilizando habitualmente analizadores cuadrupolares. Con esta técnica el modo de ionización que se aplica comúnmente es la ionización electrónica (EI), y se trabaja en monitorización selectiva de iones (SIM). El patrón de fragmentación que presentan los VMS se caracteriza principalmente por la pérdida de grupos metilo, generándose iones $[\mathrm{M}-15]^{+}$que normalmente son los que seleccionan en modo SIM. ${ }^{30}$

En cuanto a las dificultades que se pueden presentar en el análisis de estos compuestos, debe destacarse que existen dos de notable influencia: la presencia de contaminación de fondo y la ocurrencia de pérdidas por volatilización, las que pueden afectar de manera significativa la determinación cuantitativa de los VMS. ${ }^{31,32}$ Dada la importancia que tiene este tema, en esta revisión se discuten los problemas relacionados con el análisis de los VMS, y se comentan diferentes estrategias que se han propuesto en la literatura para una determinación analítica adecuada de los VMS.

\section{PROBLEMÁTICA DEL ANÁLISIS DE LOS VMS}

\section{Contaminación de fondo}

Debido a la gran cantidad de materiales y productos que contienen siliconas, en la determinación de los VMS suele detectarse la presencia de estos compuestos como contaminantes de fondo en los blancos de método. Este fenómeno puede dar lugar a falsos positivos y a errores en la cuantificación. Para minimizar y evitar en lo posible esta contaminación y así obtener resultados fiables, el análisis de los VMS requiere de la aplicación de cuidados especiales, además del empleo sistemático de blancos que permitan un control riguroso de cada una de las etapas del procedimiento analítico.

Entre las principales fuentes de contaminación que se presentan en el análisis de los VMS, hay que citar los septum de silicona comúnmente utilizados en los inyectores de los cromatógrafos de gases, los que generan una contribución importante de VMS cíclicos. La estrategia aplicada por la mayoría de los autores para minimizar esta contaminación, consiste en utilizar un septum Merlin en sustitución al de silicona. ${ }^{33-38}$ Los septum Merlin están constituidos por un material elastómero a base de fluorocarbono resistente a elevadas temperaturas, y esto permite que su utilización no aporte VMS al análisis. En la Figura 2a, se muestra a modo de ejemplo la reducción alcanzada en la señal de contaminación de fondo, alrededor de un $90 \%$, al emplearse en el inyector del cromatógrafo un septum tipo Merlin en lugar de los septum de silicona Thermogreen y BTO. ${ }^{36}$

Otra de las fuentes de contaminación de fondo en el análisis de VMS lo constituye la fase estacionaria de las columnas cromatográficas, normalmente de polidimetilsiloxano, ya que pueden aportar D3 y D4 a los blancos instrumentales como consecuencia de su degradación térmica y/o oxidativa. ${ }^{30}$ Ahora bien, es importante señalar que la contaminación de fondo de VMS producida por el sangrado de las columnas es significativamente menor que el aporte de los septum de silicona. ${ }^{31}$ No obstante, con el propósito de disminuir al máximo esta contaminación, se utilizan normalmente columnas con fases estacionarias de bajo sangrado (por ejemplo: VF-5 MS, DBXLB, DB-1 MS y DB-5 MS) ya que producen un aporte de VMS muy reducido. Otra alternativa recomendada por algunos autores para disminuir esta contaminación de fondo, consiste en emplear columnas con fases estacionarias de polietilenglicol como la DB-FFAP y la DB-Wax, aunque su uso ha sido mucho menor. ${ }^{38,39}$

Además de las fuentes de contaminación que se han mencionado, existen otras que están asociadas a las etapas de pre-tratamiento y extracción de las muestras. En este caso se debe mencionar especialmente la presencia de VMS en el aire de los laboratorios, así como en los materiales y reactivos, lo que puede provocar una contaminación notable tanto de las muestras como de los disolventes y reactivos utilizados en el análisis. Así por ejemplo, algunos autores han evaluado los niveles de VMS cíclicos D4, D5 y D6 en el aire de los laboratorios encontrando concentraciones muy elevadas. De esta manera, Yucuis y col. ${ }^{40}$ detectaron una concentración de hasta $39 \mu \mathrm{g}$ $\mathrm{m}^{-3}$ de D5 en el aire de unos laboratorios de la Universidad de Iowa. Entre los focos de emisión responsables de la presencia de los VMS en el aire de los laboratorios se puede mencionar el equipamiento de laboratorio, las juntas de goma de silicona, el aceite de las bombas de vacío, así como las emisiones producidas por el personal técnico de laboratorio que realiza el análisis, debido al uso generalizado de productos de higiene personal. ${ }^{30}$

Para evitar la contaminación producida por la presencia de VMS en el aire de laboratorio se han sugerido diferentes estrategias. Una de ellas consiste en realizar el pre-tratamiento y extracción de las muestras, así como la preparación de los patrones de calibración, en el sitio de muestreo, de modo que se evita el contacto de muestras y patrones con el aire del laboratorio. ${ }^{38,41}$ Sin embargo, realizar estas etapas del análisis en el sitio de muestreo no siempre resulta práctico, y en su lugar normalmente se opta por utilizar una cámara limpia con una cabina de flujo laminar. Este procedimiento ha resultado efectivo para minimizar la contaminación de fondo en el análisis de diferentes tipos de muestras, incluyendo aire, biota y sedimentos y ha sido el empleado por la mayor parte de los autores. ${ }^{33,39,42-44}$ A modo de
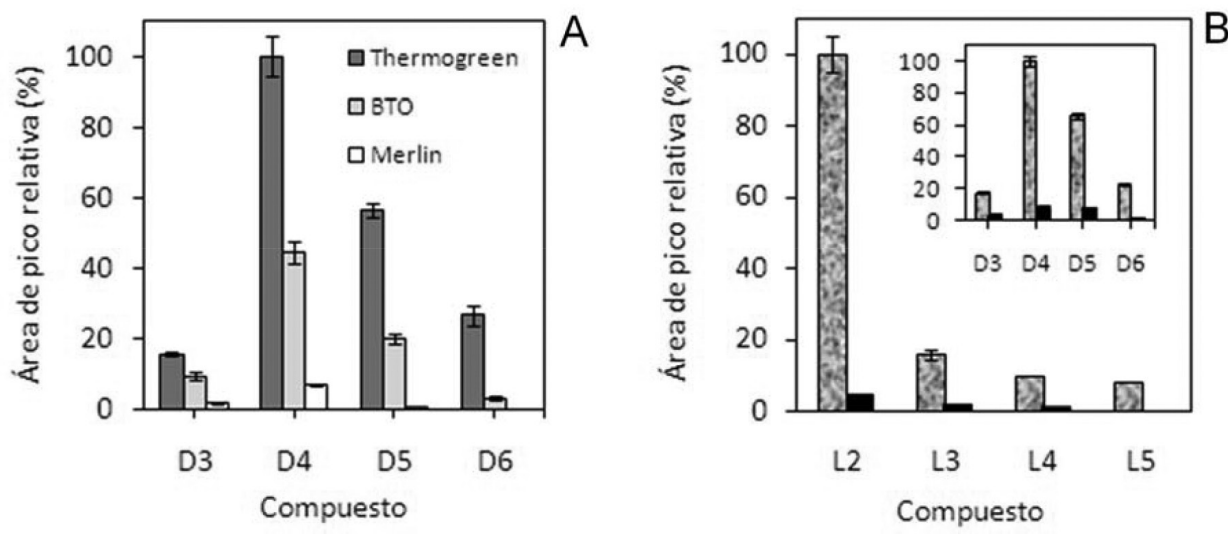

Figura 2. Contaminación de fondo detectada en el análisis de VMS en agua mediante HS-SPME utilizando en el inyector distintos tipos de septum (A): Thermo-

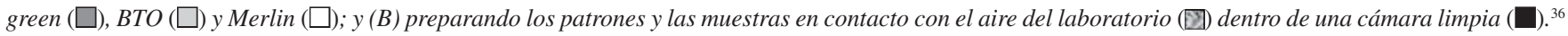


ejemplo, en la Figura $2 \mathrm{~b}$ se muestra cómo se reduce la contaminación de fondo, en un 94\%, en el análisis de VMS en agua superficial, al utilizar una cámara limpia para realizar la preparación de patrones y el pre-tratamiento de muestras. ${ }^{36,37}$

Como mencionamos anteriormente, la contaminación de fondo también puede deberse a la presencia de VMS en los materiales y reactivos de laboratorio que se emplean en el análisis. Así como ejemplo podemos mencionar que se ha detectado la presencia de D5 en cartuchos de extracción en fase sólida (SPE) Isolute ENVI+, utilizados para la toma de las muestras de aire. ${ }^{42}$ De la misma manera, Sparham y col. ${ }^{38}$ observaron una contaminación importante por D5 en el agua MilliQ utilizada como blanco para la preparación de los patrones de calibración. Estos dos ejemplos demuestran, que es imprescindible realizar un control riguroso del material de laboratorio a emplear durante el análisis, para evitar que se produzca contaminación. De igual forma, también se conoce que la contaminación de fondo puede deberse a los VMS emitidos por el técnico que realiza el análisis, ya que los productos de higiene personal que se utilizan habitualmente contienen elevadas concentraciones de VMS, especialmente de D4, D5 y D6. ${ }^{31,45,46}$ Para evitar este tipo de contaminación, los protocolos de análisis de VMS indican que los técnicos y analistas no deben utilizar productos de higiene personal durante el muestreo y análisis. ${ }^{12,39,41}$

Por último, hay que mencionar que en el control de la contaminación de fondo también debe prestar una atención especial a la etapa de toma de muestra, ya que éstas se pueden contaminar por la deposición atmosférica de VMS en el sitio de muestreo. Para controlar esta contaminación la práctica común es el empleo de blancos de muestreo. Sin embargo, la obtención y preparación de muestras blancas para el análisis de VMS también presenta dificultades, pues dada la presencia ubicua de estos compuestos, se hace difícil en algunos casos disponer de una muestra que no los contenga. Además, no siempre es posible preparar una muestra blanca artificial que simule bien la matriz analizada. Por estas razones se ha reportado el uso de muestras blancas muy variadas, como es el caso de Bletsou y col. ${ }^{47}$ que emplearon muestras de suelos que no contenían niveles detectables de VMS, como matriz blanca en el análisis de fangos de depuradora. Como otro ejemplo podemos citar a Kaj y col. ${ }^{11,12}$ que utilizan sílice como matriz blanca para el control de la toma de muestra en análisis de suelos, fangos y sedimentos. Así mismo, se ha utilizado grasa de cerdo homogeneizada como blanco de muestreo para la determinación de D4, D5 y D6 en hígado de peces y grasa de focas, ${ }^{39,48}$ y el adsorbente Isolute ENVI+ $(\sim 60 \mathrm{mg})$ se ha empleado como blanco de muestreo en el análisis de D4, D5 y D6 en zooplancton y peces. ${ }^{2,41}$ Debe decirse que todas estas alternativas pueden ser aceptables para la preparación de muestras blancas para el análisis de VMS, siempre y cuando las mismas permitan simular de manera suficientemente adecuada el comportamiento de las muestras reales durante el muestreo y el análisis.

Ahora bien, es importante comentar que en el análisis de los VMS, en especial en el caso de los VMS cíclicos, suele obtenerse una señal de contaminación de fondo aunque se tomen medidas para su minimización. Por este motivo, la señal de la contaminación de fondo debe ser considerada a la hora de calcular los límites de detección (LODs) y cuantificación (LOQs) del método, a fin de evitar la obtención de falsos positivos. La señal de contaminación de fondo se puede determinar para cada compuesto, a partir del análisis de un número suficientemente elevado de blancos de muestreo $(n>20)$. De aquí, los valores de LODs y LOQs se calculan sumando a esta señal 3 o 10 veces su desviación estándar, respectivamente. ${ }^{33,44}$ Este procedimiento permite tener en cuenta los contaminantes que se pueden incorporar durante las diferentes etapas de análisis, desde la toma de muestra hasta la determinación final. De igual forma, para la cuantificación normalmente se utiliza la estrategia de restar del área del pico de cada compuesto en la muestra, su área correspondiente en el blanco de muestreo, y así se consigue eliminar la contribución de la contaminación de fondo.

\section{Pérdidas por volatilización}

Como se ha mencionado anteriormente, los VMS son compuestos que presentan una elevada presión de vapor, por lo que pueden sufrir pérdidas por volatilización durante el análisis y esto hace que su determinación cuantitativa conlleve serias dificultades. Estas pérdidas pueden producirse en diferentes etapas del procedimiento analítico como son: toma de muestra, conservación y almacenamiento, pre-tratamiento, extracción y pre-concentración. Para evitar estas pérdidas, se aconseja aplicar una serie de cuidados especiales entre los que podemos citar: mantener las muestras refrigeradas y en recipientes herméticos previo al análisis, reducir el tiempo de almacenaje al menor posible, y realizar la mínima manipulación durante la toma, pretratamiento y extracción de las muestras.

También en relación a las pérdidas por volatilización está el tipo de método empleado para el análisis, los cuales se muestran resumidos en la Tabla 1. Como puede notarse, algunos autores aplican técnicas de extracción y pre-concentración específicas para compuestos volátiles, tales como espacio de cabeza (HS) ${ }^{38,49}$ y purga y trampa (P\&T). ${ }^{1,2,4-6,11-13,41}$ Estas técnicas permiten realizar la extracción/pre-concentración de la muestra en una sola etapa y en viales herméticos, lo que reduce la manipulación al mínimo y en consecuencia evita las pérdidas por volatilización. Entre los trabajos en los que se ha utilizado la técnica HS se pueden citar los de Sparham y col. ${ }^{38}$ y Van Egmond y col., ${ }^{49}$ donde se aplica para el análisis de VMS cíclicos en agua superficial de río y aguas residuales. Estos autores realizan la extracción de la muestra a $80^{\circ} \mathrm{C}$ durante $10 \mathrm{~min}$, e inyectan una alícuota del espacio de cabeza (3 mL) en el GC-MS. El LOQ alcanzado para el D5 en aguas superficiales es de $10 \mathrm{ng} \mathrm{L}^{-1} \cdot{ }^{38}$ Por otra parte, la técnica del espacio de cabeza también ha sido utilizada conjuntamente con la microextraccción en fase sólida (HS-SPME) para determinar VMS lineales y cíclicos en aguas superficiales.$^{50,51}$ La fibra de polidimetilsiloxano/divinilbenceno (PDMS/DVB) ha demostrado tener una mayor capacidad para la adsorción de estos compuestos en comparación a las otras fibras disponibles comercialmente. Este método resulta efectivo para el análisis de agua superficial de río alcanzándose LODs entre 0.01 y $11 \mathrm{ng} \mathrm{L}^{-1}$ para VMS lineales y cíclicos. ${ }^{50} \mathrm{Sin}$ embargo para el análisis de aguas residuales presenta problemas a causa del efecto matriz.

La técnica P\&T también se ha aplicado en la determinación de VMS lineales y cíclicos en muestras de agua (ver tabla 1). En algunos métodos se utiliza esta técnica combinada con desorción térmica empleando Tenax (250 mg) como adsorbente. ${ }^{11,12,13} \mathrm{Su}$ aplicación también se ha extendido al análisis de VMS en fangos y sedimentos, añadiendo en este caso agua para obtener una suspensión del sólido que permita la extracción de los analitos. Los LODs alcanzados para el análisis de agua se encuentran en un rango entre $<6-100 \mathrm{ng} \mathrm{L}^{-1},{ }^{12}$ los que resultan mayores que los obtenidos con las técnicas HS (D5: $\left.6 \mathrm{ng} \mathrm{L}^{-1}\right)^{38}$ y HS-SPME (L2-L5 y D3-D6: 0.01 - 11ng L-1) ${ }^{49-51}$ (tabla 1). De igual forma, la técnica P\&T se ha aplicado en el análisis de VMS cíclicos en biota, y en este caso también se utiliza la adición de agua a la muestra para obtener una suspensión del sólido., ${ }^{1,241,52}$ A diferencia del método de P\&T aplicado a aguas, para el análisis de biota el adsorbente propuesto es el Isolute ENVI+ (50mg) o el Carbotrap B, ya que éstos permiten extraer un mayor volumen de aire que el Tenax. En este caso, la desventaja de este método es que requiere un tiempo de extracción extremadamente largo (24-72 h), y esto se debe a que la complejidad de una matriz de tipo biológico dificulta la transferencia de los analitos a la fase gas, y por tanto su aplicación resulta poco práctica. 
Tabla 1. Métodos de análisis de los VMS lineales y cíclicos en muestras de suelos, sedimentos, lodos, biota y aguas mediante GC-MS

\begin{tabular}{|c|c|c|c|c|c|c|}
\hline Extracción / preconcentr. & Método de análisis & Analitos & Matriz & $\begin{array}{c}\text { Recuperación } \\
(\%) \\
\end{array}$ & LODs & Referencias \\
\hline \multicolumn{7}{|l|}{ Sedimentos, lodos y suelos } \\
\hline $\begin{array}{l}\text { Extracción con agitación } \\
\text { (n-Hex) }\end{array}$ & $\begin{array}{c}\text { GC-HRMS EI }{ }^{+}, \text {DB-Wax ETR } \\
(30 \mathrm{~m} \times 0,25 \mathrm{~mm} \times 0,25 \mu \mathrm{m})\end{array}$ & D4-D6 & sedimentos & - & 0,6-0,9 ng g-1 p.h. & 54 \\
\hline $\begin{array}{l}\text { Extracción con agitación } \\
\text { (n-Hex) }\end{array}$ & $\begin{array}{c}\text { GC-FID, VF-1MS } \\
(30 \mathrm{~m} \times 0,25 \mathrm{~mm} \times 0,25 \mu \mathrm{m})\end{array}$ & D4, D5 & fangos activados & 74-101 & - & 53 \\
\hline $\begin{array}{l}\text { Extracción con agitación } \\
(\mathrm{n}-\mathrm{Hex})\end{array}$ & $\begin{array}{l}\text { CSR-LVI-GC-MS EI+ }{ }^{+} \text {HP-5 MS } \\
\quad(60 \mathrm{~m} \times 0,25 \mathrm{~mm} \times 0,25 \mu \mathrm{m})\end{array}$ & L2-L5, D3-D6 & $\begin{array}{l}\text { fangos activados, } \\
\text { suelos }\end{array}$ & $80-103$ & $\begin{array}{l}\text { LOQs: } 0,01-1,5 \mathrm{ng} \\
\mathrm{g}^{-1} \text { p.s. }\end{array}$ & 44 \\
\hline $\begin{array}{l}\text { Extracción con agitación } \\
\text { (n-Hex, DCM/n-Hex 1:1, } \\
\text { acetato de etilo/n-Hex, } \\
1: 1, \mathrm{v} / \mathrm{v})\end{array}$ & $\begin{array}{c}\text { GC-MS EI+ }, \text { HP-5 MS } \\
(30 \mathrm{~m} \times 0,25 \mathrm{~mm} \times 0,25 \mu \mathrm{m})\end{array}$ & L3-L5, D4-D6 & fangos activados & $49-71^{*}$ & 0,003-3,3 ng g ${ }^{-1}$ p.s. & 47 \\
\hline $\begin{array}{l}\text { Extracción con agitación } \\
\text { (acetato de etilo/n-Hex. 1:1, } \\
\text { v/v), purificación : sílice }\end{array}$ & $\begin{array}{c}\text { GC-MS EI+, Rxi-5 MS } \\
(30 \mathrm{~m} \times 0,25 \mathrm{~mm} \times 0,25 \mu \mathrm{m})\end{array}$ & L4, L5, D4-D6 & $\begin{array}{l}\text { Sedimentos, fan- } \\
\text { gos activados }\end{array}$ & $\begin{array}{l}\text { Valor promedio: } \\
79^{*}\end{array}$ & $\begin{array}{l}\text { LOQs: } 0,28-2,0 \mathrm{ng} \\
\mathrm{g}^{-1} \text { p.s. }\end{array}$ & 56 \\
\hline $\begin{array}{l}\text { Extracción con agitación } \\
\text { (ACN/n-Pentano, 1:1, v/v) }\end{array}$ & $\begin{array}{c}\text { GC-MS EI }{ }^{+}, \text {HP-5 MS } \\
(30 \mathrm{~m} \times 0,25 \mathrm{~mm} \times 0,25 \mu \mathrm{m}) \\
\text { PTV-LVI }(15 \mu \mathrm{L})\end{array}$ & D4-D6 & $\begin{array}{l}\text { suelos, sedi- } \\
\text { mentos, fangos } \\
\text { activados }\end{array}$ & $69-114$ & 3-11ng g-1p.s. & 55 \\
\hline $\begin{array}{l}\text { UAE (acetato de etilo/ } \\
\text { n-Hex, } 1: 1, \mathrm{v} / \mathrm{v})\end{array}$ & $\begin{array}{c}\text { GC-MS/MS EI+, DB-5 MS } \\
(30 \mathrm{~m} \times 0,25 \mathrm{~mm} \times 0,25 \mu \mathrm{m})\end{array}$ & L3-L5, D3-D5 & sedimentos & $82-108$ & $0,3-0,9$ ng g $^{-1}$ p.s. & 59 \\
\hline $\begin{array}{l}\text { UAE (acetona) + HS-SPME } \\
\left(\mathrm{PDMS} / \mathrm{DVB}, 25^{\circ} \mathrm{C}, 40 \mathrm{~min}\right)\end{array}$ & $\begin{array}{c}\text { GC-MS EI+ }{ }^{+} \text {HP-5 MS } \\
(30 \mathrm{~m} \times 0,25 \mathrm{~mm} \times 0,25 \mu \mathrm{m})\end{array}$ & L4, L5, D3-D6 & fangos activados & $75-93^{*}$ & $<1 \mathrm{ng} \mathrm{g}^{-1} \mathrm{ps}$ & 51 \\
\hline $\begin{array}{l}\text { Extracción en columna } \\
\text { (UAE) (n-Hex) }\end{array}$ & $\begin{array}{c}\text { GC-MS EI+ }{ }^{+} \text {ZB-5 MS } \\
(30 \mathrm{~m} \times 0.25 \mathrm{~mm} \times 0.10 \mu \mathrm{m})\end{array}$ & L4-L5, D4-D6 & suelos & $88-111^{*}$ & $0,4-1,1 \mathrm{ng} \mathrm{g}^{-1} \mathrm{p} . \mathrm{s}$ & 60 \\
\hline $\begin{array}{l}\text { PLE (acetato de etilo, } 100{ }^{\circ} \mathrm{C} \\
\text { y } 1500 \text { psi) } \\
\text { Extracción con agitación } \\
(\text { ACN + n-Hex) }\end{array}$ & $\begin{array}{c}\text { GC-MS EI }, \text { ZB-5HT } \\
(30 \mathrm{~m} \times 0,25 \mathrm{~mm} \times 0,25 \mu \mathrm{m}) \\
\text { On-column }(5 \mu \mathrm{L})\end{array}$ & $\begin{array}{l}\text { D4, D5 } \\
\text { D5 }\end{array}$ & sedimentos & $\begin{array}{r}73-78 \\
85,113\end{array}$ & $\begin{array}{l}\text { 7-37 ng g }{ }^{-1} \text { p.s. } \\
1 \text { ng g-1}^{-1} \text { p.s. }\end{array}$ & 43 \\
\hline $\begin{array}{l}\text { Purga y trampa (Tenax, } \\
\left.70^{\circ} \mathrm{C}, 20 \mathrm{~min}\right)\end{array}$ & $\begin{array}{c}\text { TD-GC- HRMS EI+ } \\
\text { CP-SIL8CB } \\
(30 \mathrm{~m} \times 0,25 \mathrm{~mm} \times 0,5 \mu \mathrm{m})\end{array}$ & L2-L5, D3-D6. & $\begin{array}{l}\text { sedimentos, } \\
\text { fangos } \\
\text { activados }\end{array}$ & - & $0,3-50 \operatorname{ng~g}^{-1} \mathrm{p} . \mathrm{h}$ & 11,12 \\
\hline \multicolumn{7}{|l|}{ Biota marina } \\
\hline $\begin{array}{l}\text { Extracción con agitación } \\
\text { (n-Hex) }\end{array}$ & $\begin{array}{c}\text { GC- HRMS EI } \\
\text { HP-Ultra2 } \\
(25 \mathrm{~m} \times 0,2 \mathrm{~mm} \times 0,11 \mathrm{~mm})\end{array}$ & $\begin{array}{l}\text { L2-L5, } \\
\text { D3-D6. }\end{array}$ & peces y focas & - & $0,4-5 \mathrm{ng} \mathrm{g}^{-1}$ p.h. & 11,12 \\
\hline $\begin{array}{l}\text { Extracción con agitación } \\
\text { (n-Hex) }\end{array}$ & $\begin{array}{c}\text { GC- HRMS EI } \\
\text { HP-Ultra2 } \\
(25 \mathrm{~m} \times 0,2 \mathrm{~mm} \times 0,11 \mathrm{~mm}) \\
\end{array}$ & L3-L5, D3-D6. & $\begin{array}{l}\text { peces y meji- } \\
\text { llones }\end{array}$ & - & 0,04-50 ng g-1 p.h. & 13 \\
\hline $\begin{array}{l}\text { Extracción con agitación } \\
(\mathrm{n}-\mathrm{Hex})\end{array}$ & $\begin{array}{c}\text { GC- HRMS EI } \\
\text { HP-Ultra2 } \\
(25 \mathrm{~m} \times 0,2 \mathrm{~mm} \times 0,11 \mathrm{~mm})\end{array}$ & L3-L5, D3- D6. & peces & - & 0,09-11 ng g ${ }^{-1}$ p.h. & 58 \\
\hline $\begin{array}{l}\text { Extracción con agitación } \\
\text { (n-Hex) }\end{array}$ & $\begin{array}{c}\text { GC-HRMS EI } \\
\text { DB-Wax ETR } \\
(30 \mathrm{~m} \times 0,25 \mathrm{~mm} \times 0,25 \mu \mathrm{m})\end{array}$ & D4-D6 & $\begin{array}{l}\text { peces, focas y } \\
\text { zooplancton }\end{array}$ & $83-91$ & $0,5-10,1 \mathrm{ng} \mathrm{g}^{-1} \mathrm{p} . \mathrm{h}$. & 39 \\
\hline $\begin{array}{l}\text { Extracción con agitación } \\
\text { (THF) }\end{array}$ & $\begin{array}{c}\text { GC-MS EI+ }{ }^{+} \text {HP-5 MS } \\
(30 \mathrm{~m} \times 0,25 \mathrm{~mm} \times 0,25 \mu \mathrm{m})\end{array}$ & D4, D5 & peces & $82-93$ & 30-100 ng g-1 p.h. & 57 \\
\hline $\begin{array}{l}\text { Extracción con agitación } \\
\text { (n-Pentano) }\end{array}$ & $\begin{array}{c}\text { GC-MS EI }{ }^{+}, \text {HP-5 MS } \\
(30 \mathrm{~m} \times 0,25 \mathrm{~mm} \times 0,25 \mu \mathrm{m}) \\
\text { PTV-LVI }(15 \mu \mathrm{l}) \\
\end{array}$ & D4-D6 & peces & $70-91$ & 25-39 ng g ${ }^{-1}$ p.h. & 55 \\
\hline 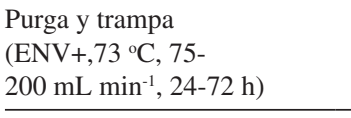 & $\begin{array}{c}\text { GC-MS EI }{ }^{+}, \text {DB-5 MS } \\
(30 \mathrm{~m} \times 0,25 \mathrm{~mm} \times 0,25 \mu \mathrm{m})\end{array}$ & D4-D6 & peces y focas & $56-82$ & $0,21-0,56$ ng g g$^{-1}$ p.h. & $2,4,5,52$ \\
\hline $\begin{array}{l}\text { Purga y trampa }\left(\mathrm{ENV}+, 73{ }^{\circ} \mathrm{C},\right. \\
\left.75-200 \mathrm{~mL} \mathrm{~min}^{-1}, 36 \mathrm{~h}\right)\end{array}$ & $\begin{array}{c}\text { GC-MS EI+ }{ }^{+} \text {DB-5 MS } \\
(30 \mathrm{~m} \times 0,25 \mathrm{~mm} \times 0,25 \mu \mathrm{m})\end{array}$ & D4-D6 & $\begin{array}{l}\text { peces y zoo- } \\
\text { plancton }\end{array}$ & - & $\begin{array}{l}\text { LOQs: 0,42-3,7 ng } \\
\text { g-1 p.h. }\end{array}$ & 41 \\
\hline $\begin{array}{l}\text { Purga y trampa (Carbotrap B, } \\
\left.75^{\circ} \mathrm{C}, 100 \mathrm{~mL} \mathrm{~min}^{-1}, 15 \mathrm{~min}\right)\end{array}$ & $\begin{array}{c}\text { TD-GC-MS EI+, DB-5 MS } \\
(30 \mathrm{~m} \times 0,25 \mathrm{~mm} \times 0,25 \mu \mathrm{m})\end{array}$ & D4-D6 & peces & $19-117$ & $2-7 \mathrm{ng} \mathrm{g}^{-1} \mathrm{p} \cdot \mathrm{h}$ & 1 \\
\hline
\end{tabular}


Tabla 1. Métodos de análisis de los VMS lineales y cíclicos en muestras de suelos, sedimentos, lodos, biota y aguas mediante GC-MS (cont.)

\begin{tabular}{|c|c|c|c|c|c|c|}
\hline Extracción / preconcentr. & Método de análisis & Analitos & Matriz & $\begin{array}{l}\text { Recuperación } \\
(\%)\end{array}$ & LODs & Referencias \\
\hline \multicolumn{7}{|l|}{ Aguas } \\
\hline $\begin{array}{l}\text { líquido-líquido (n-Hex, } \\
\text { DCM/n- Hex/, 1:1, acetato } \\
\text { de etilo/n-Hex, 1:1,v/v) }\end{array}$ & $\begin{array}{c}\text { GC-MS EI }{ }^{+}, \mathrm{HP}-5 \mathrm{MS} \\
(30 \mathrm{~m} \times 0,25 \mathrm{~mm} \times 0,25 \mu \mathrm{m})\end{array}$ & L3-L5, D4-D6 & aguas residuales & $49-108^{*}$ & $0,03-8,1 \mathrm{ng} \mathrm{L}^{-1}$ & 47 \\
\hline líquido-líquido (n-Hex) & $\begin{array}{c}\text { GC-MS/MS EI } \\
\text { DB-5 MS } \\
(30 \mathrm{~m} \times 0,25 \mathrm{~mm} \times 0,25 \mu \mathrm{m})\end{array}$ & L3- L5, D3-D5 & $\begin{array}{l}\text { agua de río, } \\
\text { aguas residuales }\end{array}$ & $40-115^{*}$ & $0,1-13 \mathrm{ng} \mathrm{L}^{-1}$ & 59 \\
\hline $\begin{array}{l}\text { MASE (n-Pentano, } 29^{\circ} \mathrm{C} \text {, } \\
60 \mathrm{~min} \text { ) }\end{array}$ & $\begin{array}{c}\text { GC-MS EI }{ }^{+}, \mathrm{HP}-5 \mathrm{MS} \\
(30 \mathrm{~m} \times 0,25 \mathrm{~mm} \times 0,25 \mu \mathrm{m}) \\
\text { PTV-LVI }(15 \mu \mathrm{L})\end{array}$ & D4-D6 & $\begin{array}{l}\text { agua de río, } \\
\text { aguas residuales }\end{array}$ & $100-107$ & 9-27 ng L-1 & 55 \\
\hline $\begin{array}{l}\text { UAE-DLLME (clorobence- } \\
\text { no, } 13 \mu \mathrm{L} / 13 \mathrm{~mL}, 2300 \mathrm{rpm} \text { ) }\end{array}$ & $\begin{array}{c}\text { GC-MS EI+, DB-624 } \\
(60 \mathrm{~m} \times 0,25 \mathrm{~mm} \times 1,4 \mu \mathrm{m})\end{array}$ & $\begin{array}{l}\text { L2, L4, L5, } \\
\text { D3-D6 }\end{array}$ & aguas residuales & $71-99$ & $2-400 \mathrm{ng} \mathrm{L}^{-1}$ & 61 \\
\hline $\mathrm{HS}\left(80^{\circ} \mathrm{C}, 10 \mathrm{~min}\right)$ & $\begin{array}{c}\text { GC-MS EI }, \text { DB-FFAP } \\
(30 \mathrm{~m} \times 0,25 \mathrm{~mm} \times 0,25 \mu \mathrm{m})\end{array}$ & D5 & $\begin{array}{l}\text { agua de río, } \\
\text { aguas residuales }\end{array}$ & $81-99$ & LOQs: $10 \mathrm{ng} \mathrm{L}^{-1}$ & 38 \\
\hline $\mathrm{HS}\left(80^{\circ} \mathrm{C}, 10 \mathrm{~min}\right)$ & $\begin{array}{c}\text { GC-MS EI }{ }^{+} \text {, DB-Wax }(30 \mathrm{~m} \times 0,25 \mathrm{~mm} \\
\times 0,25 \mu \mathrm{m})\end{array}$ & D4-D6 & aguas residuales & $14-98$ & $10-200 \mathrm{ng} \mathrm{L}^{-1}$ & 49 \\
\hline $\begin{array}{l}\text { HS-SPME (PDMS/DVB, } \\
\left.25^{\circ} \mathrm{C}, 40 \mathrm{~min}\right)\end{array}$ & $\begin{array}{c}\text { GC-MS EI }{ }^{+}, \mathrm{HP}-5 \mathrm{MS} \\
(60 \mathrm{~m} \times 0,25 \mathrm{~mm} \times 0,25 \mu \mathrm{m})\end{array}$ & L2- L5, D3-D6 & agua de río & & $0,01-11 \mathrm{ng} \mathrm{L}^{-1}$ & 50 \\
\hline $\begin{array}{l}\text { HS-SPME (PDMS/DVB, } \\
\left.25^{\circ} \mathrm{C}, 40 \mathrm{~min}\right)\end{array}$ & $\begin{array}{c}\text { GC-MS EI }, \text { HP-5 MS } \\
(30 \mathrm{~m} \times 0,25 \mathrm{~mm} \times 0,25 \mu \mathrm{m})\end{array}$ & L4, L5, D3-D6 & aguas residuales & $78-96$ & $3,2-7,8 \mathrm{ng} \mathrm{L}^{-1}$ & 51 \\
\hline $\begin{array}{l}\text { Purga y trampa (Tenax, } \\
\left.70^{\circ} \mathrm{C}, 20 \mathrm{~min}\right)\end{array}$ & $\begin{array}{c}\text { TD-GC- HRMS EI+ } \\
\text { CP-SIL8CB } \\
(30 \mathrm{~m} \times 0,25 \mathrm{~mm} \times 0,5 \mu \mathrm{m})\end{array}$ & L2-L5, D3-D6. & $\begin{array}{l}\text { agua de mar y } \\
\text { lagos, aguas } \\
\text { residuales }\end{array}$ & - & $<6-100 \mathrm{ng} \mathrm{L}^{-1}$ & 11,12 \\
\hline $\begin{array}{l}\text { Purga y trampa (Tenax, } \\
\left.70^{\circ} \mathrm{C}, 20 \mathrm{~min}\right)\end{array}$ & $\begin{array}{c}\text { TD-GC- HRMS EI+ } \\
\text { CP-SIL8CB } \\
(30 \mathrm{~m} \times 0,25 \mathrm{~mm} \times 0,5 \mu \mathrm{m})\end{array}$ & L3-L5, D4-D6. & aguas residuales & - & & 13 \\
\hline
\end{tabular}

*: Métodos que aplican etapas de concentración con rotavapor o con corriente de nitrógeno. ACN: acetonitrilo; DCM: diclorometano; n-Hex: n-hexano; CSR-LVI: Concurrent solvent recondensation - Large volumen injection; P\&T: Purga y trampa; THF: tetrahidrofurano; LODs: Límites de detección del método; LOQs: Límites de cuantificación del método; PUF: Espuma de Poliuretano; UAE: Extracción asistida por ultrasonidos; PLE: Extracción con líquidos presurizados; MASE: Extracción con disolventes asistida por membranas; SPE: Extracción en fase sólida; HS: Extracción en espacio de cabeza; HS-SPME: Microextracción en fase sólida del espacio de cabeza; TD-GC-MS: Desorción térmica combinada con cromatografía de gases y espectrometría de masas, p.s.: peso seco, p.h.: peso húmedo, MS/MS: espectrometría de masas en tándem.

A pesar de las ventajas que brinda la aplicación de las técnicas de extracción/pre-concentración HS, HS-SPME y P\&T para determinar VMS, en el caso de analizar de matrices complejas como aguas residuales y biota, donde el efecto matriz puede ser importante, con frecuencia se opta por aplicar la extracción con disolventes. Sin embargo, la desventaja que conlleva utilizar la extracción con disolvente de estos compuestos volátiles, es que pueden producirse pérdidas por volatilización. Para evitar estas pérdidas la práctica común consiste en utilizar procedimientos de extracción sencillos y rápidos, no aplicar etapas de concentración a los extractos, sea con rotavapor o con flujo de nitrógeno, y evitar en lo posible el uso de etapas de purificación.

Los métodos publicados en la literatura que utilizan la extracción con disolventes se muestran en la tabla 1. Como puede notarse, para el caso de matrices sólidas la extracción con disolvente se realiza, por lo común, aplicando agitación utilizando un vortex. Los disolventes comúnmente utilizados son: n-hexano, n-pentano, acetonitrilo, clorobenceno, y mezclas acetato de etilo/n-hexano y diclorometano/n-hexano ${ }^{11-13,39,44,48,55-59}$. Como alternativa a la extracción con disolvente mediante agitación, también se ha aplicado la extracción asistida por ultrasonidos (UAE) ${ }^{51,59,60}$ y la extracción con líquidos presurizados (PLE) ${ }^{43}$ sin embargo, estos métodos no son recomendables dado el hecho de que pueden producirse pérdidas de los analitos más volátiles al utilizarse elevadas temperaturas y presión. Debe decirse que de manera general, en los métodos que aplican la extracción con disolventes, se han logrado alcanzar recuperaciones superiores al $70 \%$ y LODs entre 0.003 y $37 \mathrm{ng} \mathrm{g}^{-1}$ p.s. (tabla 1 ). Sin embargo, es importante destacar que en la mayor parte de estos métodos se inyecta el extracto directamente en el cromatógrafo de gases (GC) sin una etapa previa de purificación, con la idea de simplificar así el procedimiento de análisis para evitar pérdidas por volatilización. Como ejemplo podemos citar el método reportado por Warner y col. ${ }^{39}$ para determinar D4, D5 y D6 en muestras de pescado, en el cual la extracción se realiza mediante agitación con n-hexano y luego se procede a inyectar directamente el extracto en el GC. Como se conoce, la inyección directa de extractos comprende un riesgo importante que puede ocasionar contaminación en el sistema de inyección. Una solución a este problema es la propuesta por Companioni-Damas y col. ${ }^{44}$ en un método utilizado para el análisis de suelos y fangos, donde se utiliza un cartucho de SPE de sílice $(100 \mathrm{mg} / 1 \mathrm{~mL})$, para purificar el extracto orgánico obtenido previo al análisis en el GC.

La extracción con disolventes también se ha aplicado al análisis de VMS en matrices líquidas. Así por ejemplo, se ha utilizado la microextracción con disolventes asistida por membrana (MASE) para determinar VMS en aguas residuales. ${ }^{55}$ En este método se utiliza una membrana de polietileno, una temperatura y tiempo de extracción de $29^{\circ} \mathrm{C}$ y $60 \mathrm{~min}$, y como disolvente se utilizó el n-pentano. Para determinar VMS en aguas residuales también se ha aplicado la microextracción líquido-líquido dispersiva asistida por ultrasonidos (UAE-DLLME) ${ }^{61}$ En este caso se utiliza el clorobenceno como disolvente de extracción y una relación volumen de disolvente/ 
volumen de muestra de $13 \mu \mathrm{L} / 13 \mathrm{~mL}$. Así, en el análisis de VMS en aguas residuales, tanto la extracción MASE como la UAE-DLLME permiten alcanzar recuperaciones superiores al 70\% y LODs en un intervalo entre 2 y $400 \mathrm{ng} \mathrm{L}^{-1}$ (tabla 1). Por último, se debe mencionar que también se ha aplicado la extracción líquido-líquido convencional en el análisis de VMS en agua residual ${ }^{47,59}$ Sin embargo, esta opción no se recomienda ya que puede ocasionar pérdidas significativas de los compuestos más volátiles, y en su lugar es preferible aplicar las técnicas de microextracción ya citadas.

Como hemos mencionado anteriormente, para minimizar las pérdidas por volatilización durante el análisis de VMS se suele evitar el uso de etapas de pre-concentración de los extractos, sin embargo, esto conlleva una pérdida de sensibilidad importante en la determinación. Para compensar esta pérdida de sensibilidad algunos autores utilizan la inyección de grandes volúmenes en el GC. Así por ejemplo Wang y col. ${ }^{55}$ utilizaron la inyección de grandes volúmenes con vaporización a temperatura programada (PTV-LVI), en el modo de derivación del disolvente (solvent split). Con esta técnica alcanzaron inyectar hasta $15 \mu \mathrm{l}$ de extracto (en n-pentano) y así obtener, para el análisis de D4, D5 y D6 en suelos, sedimentos y fangos, LODs entre 3 y $11 \mathrm{ng} \mathrm{g}^{-1}$ peso seco (p.s.). Sin embargo, debe mencionarse que la aplicación de la inyección PTV-LVI tiene como desventaja, que requiere de una cuidadosa optimización de las condiciones experimentales de inyección, dado que estos analitos volátiles pueden perderse durante la etapa de eliminación del disolvente.

Como alternativa al uso de la inyección PTV-LVI en el análisis de VMS, se ha aplicado la inyección splitless de grandes volúmenes con recondensación concurrente de disolvente (CSR-LVI) ${ }^{44}$ En comparación con la inyección PTV-LVI, la ventaja más importante de esta técnica consiste en que la muestra inyectada se transfiere completamente a la columna cromatográfica, sin la eliminación del disolvente mediante una válvula split, con lo cual esta técnica resulta ideal para el análisis de estos compuestos volátiles. Además, la técnica CSRLVI permite inyectar un mayor volumen de extracto $(30-50 \mu \mathrm{L})^{62}$
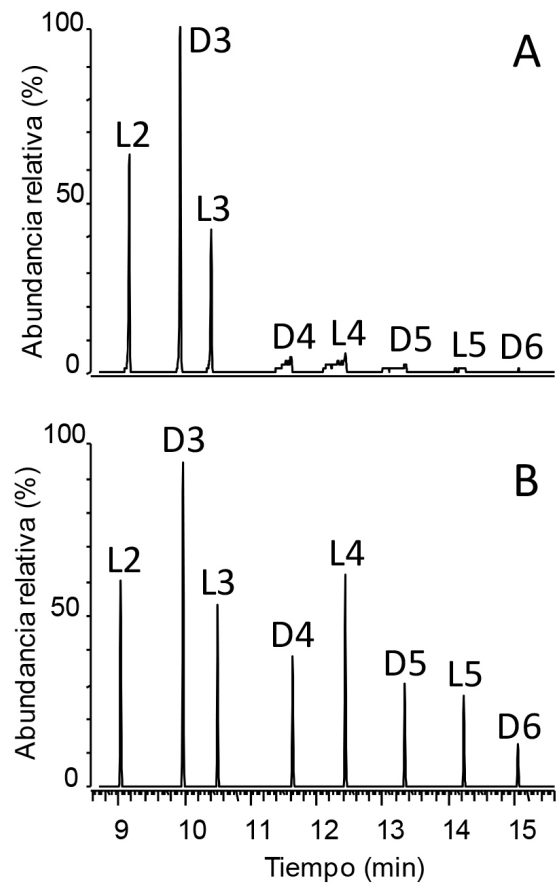

que la técnica PTV-LVI (hasta $15 \mu \mathrm{L}$ ), ${ }^{55}$ y en consecuencia se puede alcanzar un mayor incremento en sensibilidad. Así, con la aplicación de la CSR-LVI en el análisis de VMS en suelos y fangos se inyectaron $30 \mu \mathrm{L}$ de extracto orgánico (en hexano), alcanzándose LOQs (L2-L5 y D3-D6: 0.01 - $1.5 \mathrm{ng} \mathrm{g}^{-1}$ p.s. $)^{44}$, en un orden de magnitud más bajos que los reportados por Wang y col. ${ }^{55}$ utilizando la inyección PTV-LVI (D4-D6: 11 - $37 \mathrm{ng} \mathrm{g}^{-1}$ p.s.). También debe decirse que otra ventaja importante que brinda la técnica CSR-LVI, es que su implementación es relativamente sencilla, siendo los principales parámetros experimentales a optimizar el volumen y el disolvente de inyección. Así a modo de ejemplo, en la figura 3 se ilustra el efecto que tiene el disolvente de inyección en la forma de los picos cromatográficos de los VMS. Como puede observarse, tanto el diclorometano como el tetrahidrofurano y la mezcla tetrahidrofurano/n-hexano (80/20, v/v) producen picos distorsionados, efecto que se atribuye a la polaridad del disolvente de inyección, mientras que con el uso del n-hexano o una mezcla tetrahidrofurano/n-hexano (60/40, v/v) se logran obtener picos de forma óptima. Con estos dos disolventes, se logró alcanzar para los VMS un volumen máximo de inyección de 30 y $20 \mu \mathrm{L}$, respectivamente. ${ }^{36}$ Es por esta razón que la técnica CSR-LVI, dado que permite inyectar un mayor volumen de muestra y su optimización es mucho más simple, se recomienda para el análisis de VMS.

\section{CONCLUSIONES}

La contaminación de fondo, uno de los aspectos más críticos en el análisis de los VMS, puede ser minimizada si se aplican una serie de cuidados especiales en el procedimiento de análisis, como por ejemplo: emplear un septum tipo Merlin en el inyector del cromatógrafo de gases lo que permite evitar la contaminación que proviene del septum de silicona; y realizar el pre-tratamiento y extracción de las muestras, y también la preparación de los patrones de calibración en una cámara limpia, para impedir la contaminación que causa el aire de laboratorio.
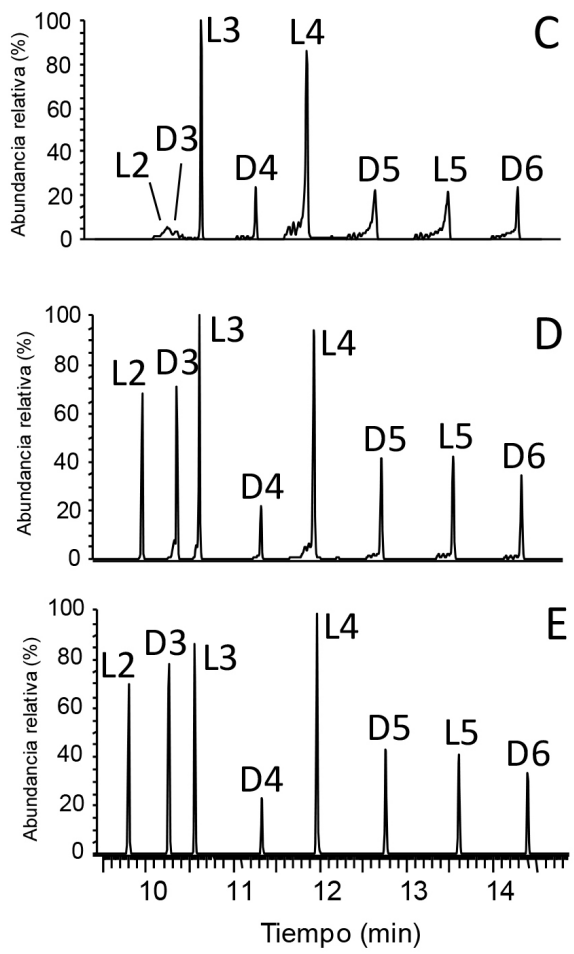

Figura 3. Efecto del disolvente de inyección en la forma de los picos obtenidos mediante CSR-LVI-GC-MS. Disolventes de inyección utilizados: (A) diclorometano, (B) n-hexano, (C) tetrahidrofurano, (D) tetrahidrofurano/n-hexano 80/20 (v/v) y (E) tetrahidrofurano/n-hexano 60/40 (v/v). Volumen de inyección:

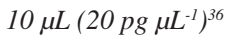


Evitar las pérdidas por volatilización es el otro aspecto importante a tener en cuenta en el análisis de VMS. La estrategia más efectiva consiste en utilizar técnicas de extracción/pre-concentración específicas para compuestos volátiles tales como: HS, HS-SPME y Purga y Trampa. Sin embargo, en presencia de un efecto matriz importante, también se puede utilizar la extracción con disolventes, aunque se deben cumplir una serie de condiciones para evitar pérdidas de volátiles como por ejemplo: emplear un tiempo de extracción mínimo (<10 min), no utilizar ni temperaturas ni presiones elevadas, y no aplicar etapas de concentración a los extractos obtenidos.

Al hacer uso de la extracción con disolventes para analizar VMS, la pérdida de sensibilidad que conlleva no concentrar los extractos orgánicos, se logra compensar haciendo uso de la inyección de grandes volúmenes en el GC. La técnica de inyección recomendada es la CSR-LVI, ya que permite inyectar un mayor volumen de muestra que la inyección PTV-LVI, no se produce el riesgo de pérdida de volátiles, y su optimización es muy simple.

\section{AGRADECIMIENTOS}

El autor está muy agradecido por el apoyo financiero del Ministerio Español de Ciencia e Innovación en el marco del Proyecto CTQ2009-09253, y también al Ministerio de Asuntos Exteriores y Cooperación de España (MEAC) y a la Agencia Española de Cooperación Internacional Cooperación (AECI), por la beca de Doctorado otorgada.

\section{REFERENCIAS}

1. McGoldrick, D. J.; Chan, C., Drouillard, K. G.; Keir, M. J.; Clark, M. G.; Backus, S. M.; Environ. Pollut. 2014, 186, 141.

2. Kierkegaard, A.; van Egmond, R.; McLachlan, M. S.; Environ. Sci. Technol. 2011, 45, 5936.

3. Warner, N. A.; Evenset, A.; Chriestensen, G.; Gabrielsen G. W.; Borga, K.; Leknes, H.; Environ. Sci. Technol. 2010, 44, 7705.

4. Kierkegaard, A.; Bignert, A.; McLachlan, M. S.; Chemosphere. 2013a, 93, 774 .

5. Kierkegaard, A.; Bignert, A.; McLachlan, M. S.; Chemosphere 2013b, 93, 789.

6. Borga, K.; Fjeld, E.; Kierkegaard, A.; McLachlan, M. S.; Environ. Sci. Technol. 2013, 47, 14394.

7. Borga, K.; Fjeld, E.; Kierkegaard, A.; McLachlan, M. S.; Environ. Sci. Technol. 2012, 46, 6347,

8. McKim, J. M.; Jr, Kolesar, G. B.; Jean, P. A.; Meeker, L. S.; Wilga, P. C.; Schoonhoven, R.; Swenberg, J. A.; Goodman, J. I.; Gallavan, R. H.; Meeks, R. G.; Toxicol. Appl. Pharmacol. 2001, 172, 83.

9. He, B.; Rhodes-Brower, S.; Miller, M. R.; Munson, A. E.; Germolec, D. R.; Walker, V. R.; Korach, K. S.; and Meade, B. J.; Toxicol. Appl. Pharmacol. 2003, 192, 254.

10. ht t p://nepis.epa.gov/exe/zy P D F.cgi/P 1004515. PDF?DOCKEYP1004515.pdf, revisada en Julio del 2016.

11. http://www.ivl.se/download/18.2f3a7b311a7c806443800055371/B1643. pdf, revisada en Marzo del 2010.

12. http://nordicscreening.org/index.php? module=Pagesetter\&type=file $\& \mathrm{f}$ unc $=$ get $\&$ tid $=5 \&$ fid=reportfile $\&$ pid $=4$, revisada en Marzo del 2010.

13. http://www.klif.no/publikasjoner/2269/ta2269.pdf, revisada en Marzo del 2013.

14. Genualdi, S.; Harner, T.; Cheng, Y.; McLead, M.; Harsen, K. M.; Van Egmond, R.; Shoeib, M.; Lee, S.; Environ. Sci. Technol. 2011, 45, 3349.

15. Xu, L.; Shi, Y.; Wang, T.; Dong, Z.; Su, W.; Cai, Y.; Environ. Sci. Technol. 2012, 46, 11718 .

16. Wang, D.-G.; Steer, H.; Tait, T.; Williams, Z.; Chemosphere 2013, 93, 766.
17. Sparham, C.; Van Egmond, R.; O’Connor, S.; Hastie, C.; Whelan, M.; Kanda, R.; Franklin, O.; J. Chromatogr. A 2008, 1212, 124.

18. Sanchís, J.; Martínez, E.; Ginebreda, A.; Farré, M.; Barceló, D.; Sci. Total Environ. 2013, 443, 530.

19. Companioni-Damas, E. Y.; Santos, F. J.; Galceran M. T.; Talanta 2012a, $89,65$.

20. Buser, A. M.; Kierkegaard, A.; Bogdal, C.; McLeod, M.; Scheringer, M.; Hungerbuhler, K.; Env. Sci. Technol. 2013, 47, 7045.

21. Kroseth, I. S.; Kierkegaard, A.; McLachlan, M. S.; Breivik, K.; Hansen, K. M.; Schlabach, M.; Env. Sci. Technol. 2013, 47, 502.

22. Varaprath, S.; Frye, C.L.; Hamelink, J; Environ. Toxicol. Chem. 1996, 15,1263 .

23. Mazzoni, S. M.; The Handbook of Environmental Chemistry. Part H. Organosilicon Materials: Eco-relevant properties of organosilicon materials, In: G. Chandra, (Ed.). Vol. 3. Springer-Verlag, Berlin, 1997, 53.

24. Lei, Y. D.; Wania, F.; Mathers, D.; J. Chem. Eng. Data 2010, 55, 5868.

25. Whelan, M. J.; Estrada, E.; van Egmond, R.; Chemosphere 2004, 57, 1427.

26. http://www.ec.gc.ca/ese-ees/13CC261E-5FB0-4D33-8000EA6C6440758A/batch2_541-02-6_en.pdf, revisada en Octubre del 2013

27. http://publications.environment-agency.gov.uk/pdf/SCHO0309BPQXe-e.pdf., revisada en marzo del 2010.

28. http://eur-lex.europa.eu/LexUriServ/LexUriServ.do?uri=oj:1:2006:396: 0001:0849:en:pdf, revisada en Octubre del 2013.

29. http://www.epa.gov/oppt/iur/tools/data/2002-vol.htm, revisada en Febrero del 2013

30. Varaprath, S.; Stutts, D. H.; Kozerski, G. E.; Silicon Chem. 2006, 3, 79.

31. Horii, Y.; Kannan, K.; Arch. Environ. Contam. Toxicol. 2008, 55, 701.

32. Warner, N. A.; Kozerski, G.; Durham, J.; Koerner, M.; Gerhards, R.; Campbell, R.; McNett, D.A.; Chemosphere 2013, 93, 749.

33. Kierkegaard, A.; McLachlan, M. S.; Atmospheric Environment 2013, 80, 322.

34. McLachlan, M. S.; Kierkegaard, A.; Hansen, K. M.; van Egmond, R.; Christensen, J. H.; Skjøth, C. A.; Environ. Sci. Technol. 2010, 44, 5365.

35. Krogseth, I. S.; Zhang, X.; Lei, Y. D.; Wania, F.; Breivik, K.; Environ. Sci. Technol. 2013, 47, 4463.

36. Companioni-Damas, E. Y.; Tesis de Doctorado, Universidad de Barcelona, España, 2014

37. Companioni-Damas, E. Y.; Santos, F. J.; Galceran M. T.; Talanta 2014 , 118, 245.

38. Sparham, C.; Van Egmond, R.; O'Connor, S.; Hastie, C.; Whelan, M.; Kanda, R.; Franklin, O.; J. Chromatogr. A 2008, 1212, 124.

39. Warner, N. A.; Evenset, A.; Christensen, G.; Gabrielsen, G. W.; Borgå, K.; Leknes, H.; Environ. Sci. Technol. 2010, 44, 7705.

40. Yucuis, R. A.; Stanier, C. O.; Hornbuckle, K. C.; Chemosphere 2013, 92, 905.

41. Borgå, K.; Fjeld, E.; Kierkegaard, A.; McLachlan, M. S.; Environ. Sci. Technol. 2012, 46, 6347.

42. Kierkegaard, A.; McLachlan, M. S.; J. Chromatogr. A 2010, 1217, 3557.

43. Sparham, C.; van Egmond, R.; Hastie, C.; O'Connor, S.; Gore, D.; Chowdhury, N.; J. Chromatogr. A 2011, 1218, 817.

44. Companioni-Damas, E. Y.; Santos, F. J.; Galceran M. T.; J. Chromatogr. A 2012a, $1268,150$.

45. Wang, R.; Moody, R. P.; Koniecki, D.; Zhu, J.; Environ. Int. 2009, 35, 900.

46. Dudzina, T.; von Goetz, N.; Bogdal, C.; Biesterbos, J. W. H.; Hungerbühler, K.; Environ. Int. 2014, 62, 86.

47. Bletsou, A. A.; Asimakopoulos, A. G.; Stasinakis, A. S.; Thomaidis, N. S.; Kannan, K.; Environ. Sci. Technol. 2013, 47, 1824.

48. Warner, N. A.; Kozerski, G.; Durham, J.; Koerner, M.; Gerhards, R.; Campbell, R.; McNett, D. A.; Chemosphere 2013, 93, 749. 
49. Van Egmond, R.; Sparham, C.; Hastie, C.; Gore, D.; Chowdhury, N.; Chemosphere 2013, 93, 757.

50. Companioni-Damas, E. Y.; Santos, F. J.; Galceran M. T.; Talanta 2012b, $89,65$.

51. Xu, L.; Shi, Y.; Cai, Y.; Water Res. 2013, 47, 715.

52. Kierkegaard, A.; Adolfsson-Erici, M.; McLachlan, M. S.; Anal. Chem. 2010, 82, 9573.

53. Borgå, K.; Fjeld, E.; Kierkegaard, A.; McLachlan, M. S.; Environ. Sci. Technol. 2013, 47, 14394.

54. Dewil, R.; Appels, L.; Baeyens, J.; Buczynska, A.; Van Vaeck, L.; Talanta 2007, 74, 14.

55. Wang, D.-G.; Alaee, M.; Steer, H.; Tait, T.; Williams, Z.; Brimble, S.; Svoboda, L.; Barresi, E.; Dejong, M.; Schachtschneider, J.; Chemosphere 2013, 93, 741.
56. Zhang, Z.; Qi, H.; Ren, N.; Li, Y.; Gao, D.; Kannan, K.; Arch. Environ. Contam. Toxicol. 2011, 60, 204.

57. Woodburn, K.; Drottar, K.; Domoradzki, J.; Durham, J.; McNett, D.; Jezowski, R.; Chemosphere 2013, 93, 779.

58. http://www.miljodirektoratet.no/old/klif/publikasjoner/2510/ta2510.pdf, revisada en Febrero del 2014.

59. Sanchís, J.; Martínez, E.; Ginebreda, A.; Farré, M.; Barceló, D.; Sci. Total Environ. 2013, 443, 530.

60. Sánchez-Brunete, C.; Miguel, E.; Albero, B.; Tadeo, J. L.; J. Chromatogr. A 2010, 1217, 7024.

61. Cortada, C.; dos Reis, L. C.; Vidal, L.; Llorca, J.; Canals, A.; Talanta 2014, 120, 191.

62. Magni, P.; Porzano, T.; J. Sep. Sci. 2003, 26, 1491. 